\title{
Turing miRNA Into Infinite Coordination Supermolecule: A General and Enabling Nanoengineering Strategy for Resurrecting Nuclear Acid Therapeutics
}

\section{Liya Li}

Xi'an Jiaotong University

Wangxiao He ( $\sim$ hewangxia05366@xjtu.edu.cn )

Xi'an Jiaotong University https://orcid.org/0000-0002-2054-6022

\section{Weiming You}

Xi'an Jiaotong University

Jin Yan

Xi'an Jiaotong University

\section{Wenjia Liu}

Xi'an Jiaotong University

\section{Research}

Keywords: miRNA clinical translation, Nuclear acid therapeutics, Nanoengineering, Infinite covalent polymer, Anti-cancer therapeutics

Posted Date: November 16th, 2021

DOI: https://doi.org/10.21203/rs.3.rs-1054917/v1

License: (c) (i) This work is licensed under a Creative Commons Attribution 4.0 International License.

Read Full License

Version of Record: A version of this preprint was published at Journal of Nanobiotechnology on January 4th, 2022. See the published version at https://doi.org/10.1186/s12951-021-01212-9. 


\section{Abstract \\ Background}

Clinical translation of therapeutic nuclear acid, particularly those targeting tumor progression, has been hampered by the intrinsic weaknesses of nuclear acid therapeutic including poor systemic stability, rapid clearance, low membrane permeability and lack of targeting ability. Small nuclear acid engineered into carrier-free nanodrugs with structural stability and disease targeting may be viable to overcome pharmaceutical obstacles of nuclear acid.

\section{Methods}

A general method through a mild and simple chemistry was established to convert therapeutic miRNA into an infinite Auric-sulfhydryl coordination supramolecular miRNA termed lacsRNA with near-spherical nanostructure, high colloid as well as anti-hydrolysis stability and low macrophage uptakes.

\section{Results}

lacsRNA presented the increased half-life period in circulation and accumulation at tumor sites in comparison to normal miRNA. Moreover, lacs-miR-30c showed no toxicity of viscera and sanguis system in the 5-time injection dosage of the treatment. More importantly, lacs-miR-30c potently suppressed the Wnt signaling pathway in vitro and in vivo, and effectively sensitized both potency of 5-Fu in PDX model of colon cancer and Anti-PD1 in B16F10 homograft model of melanoma.

\section{Conclusion}

Collectively, this work amply confirmed the design of lacsRNA as a general and viable strategy of nanopharmaceutic to concert flimsy therapeutic miRNA into potential drugs. Considering from a broader perspective, the miRNA-initiated infinite coordination self-assembly strategy has distinct advantages in resurrecting nuclear acid therapeutics, probably bringing new inspiration to RNA-derived therapeutics of a great variety of human diseases including cancer.

\section{Introduction}

MicroRNAs (miRNA), a series of 22 nucleotide-long and noncoding RNA molecules, regulate gene expression and a range of biological functions involving in the pathogenesis of a wide variety of diseases in especial of cancer [1]. In the cytoplasm, miRNAs recognize a complimentary mRNA sequence, and subsequently induce its post-translation degradation and silencing, offering great promise to create novel therapeutic approaches at transcriptional level towards down-regulating abnormally elevated pathogenesis-related proteins [2, 3]. Although a growing number of miRNAs modulating disease 
processes have been identifying, their clinical translation, particularly those targeting tumor progression, has been hampered by the intrinsic weaknesses of nuclear acid therapeutic including poor systemic stability, rapid clearance, low membrane permeability and lack of targeting ability $[1,4]$.

To alleviate these technical hurdles, various elaborate methods for miRNA modification and/or delivery have been developed, and significant progress has been made in ways of improving pharmacological potentials of miRNAs therapeutics [1]. Though chemically modified miRNAs possess the resistant against nuclease and the subsequent prolonged half-life in the bloodstream, the off-target effects severely limit their clinical application presumably because of the broad but excrescent functionality of miRNA in the healthy organs and tissues $[5,6]$. Besides, as for miRNAs delivery vehicles, the frequently-used used lipids, cationic polymers and biodegradable polymers always suffered from the rapid clearance by reticuloendothelial system (RES), in which liver and spleen macrophages eliminate these exogenous particles at a great lick from the circulation system $[7,8]$, resulting in the bereft or depressed potency of miRNAs therapeutics. Conceptually, novel and clinically viable strategies are needed to advance the clinical transformation of miRNAs.

Nanotechnology holds great promise in overcoming pharmacological weaknesses of miRNA $[9,10]$. Nanocarrier-mediated oligonucleotide delivery is capable of protecting the cargo from nuclease degradation during the circulation, and promoting oligonucleotide internalizing into cytoplasm via endocytosis that can escape endosomal degradation [11-13]. In fact, nanoparticle-based drug delivery systems are particularly attractive in the treatment of solid tumors, as nanoparticles are capable of active crossing endothelial cells into the interstitial space of the neoplasm, thereby resulting in the preferential accumulation at tumor sites [14-16]. Despite increasing success in the development of tumor-targeting nanocarriers [17-21], the overwhelming majority of nano-medicine suffered from time/cost-consuming construction, low cargo loading and improper disruption in circulation [22, 23]. Therefore, it remains a challenge to explore miRNA-derived nanotherapeutics that simultaneously possess simple synthesis, high stability, satisfying loading and excellent performance.

Carrier-free nanodrugs provided an intriguing strategy to develop nanomedicine, by which drug themselves were assembled into well-defined nanostructures through self-assembly and/or coordination $[24,25]$. Infinite coordination polymers (ICP) is an emerging class of carrier-free nanodrug system, in which drugs connect with ions directly via coordination bonds [26, 27], yielding a series of advantages including adjustable high drug loading, compositional tunability, mild preparing condition, and infinitely controllable extension in spatial dimension [26, 27]. As a consequence, we hypothesized that miRNAderived ICP can not only overcome the pharmacological weaknesses of nuclear acid therapeutics, but also possess excellent pharmaceutical superiority towards the optimized potency.

Herein, we developed a general method to convert therapeutic miRNA into a stable and bioavailable infinite Auric-sulfhydryl coordination supramolecular miRNA (lacsRNA) by a mild and simple chemistry route. In this case, auric-mercapto-miRNA precursors can self-assembled into a spherical nanostructure driven by aurophilicity (Figure 1A). As a proof of concept, miR-30c, a member of miR-30s family that 
specifically target Bcl9 in cancer[28], was used to synthesized lacsRNA (Figure 1A). As a result, lacs-miR$30 \mathrm{c}$ overcome the pharmacological obstacles of miRNA and potently suppressed the Wnt signaling pathway in vitro and in vivo, but more than that, it effectively potentiated chemotherapy and immunotherapy in vivo. This work amply confirmed the design of lacsRNA as a general and viable strategy of nano-pharmaceutic to concert therapeutic miRNA into potential drugs, thereby reinvigorating efforts for discovering nuclear acid therapeutics in a great variety of human diseases including cancer.

\section{Results}

\section{Design and Synthesis of lacsRNA}

For the construction of infinite Auric-sulfhydryl coordination polymeric miRNA (lacsRNA), an extra sulfydryl was introduced to the $5^{\prime}$ terminus of miRNA, and $\mathrm{Au}^{3+}$ in chloroauric acid was used to conjugate with the thiol to form a polymeric RNA-Au(I) compound termed [RNA-S-Au+ $]_{n}$ through infinite extension in 2D (Figure 1A), where $A u^{1+}$ ions bridge miRNA-SH by a bivalent -S-Au+-S- coordination[19, 29-31]. Subsequently, driven by the aurophilicity among $\mathrm{Au}(\mathrm{I})$, [RNA-S-Au$\left.{ }^{+}\right]_{n}$ can self-assemble into a nearspherical architecture (lacsRNA) at small nano scale [18, 30], supporting by the transmission electron microscope (TEM) image of lacsRNA (Figure 1B) and hydrodynamic diameter of lacsRNA around 38.7nm with an acceptable polymey disperse index of 0.265 measured by dynamic light scattering analysis (Figure 1C). Additionally, the surface charge of lacsRNA was -11.1 mV (Figure 1D), and this electronegativity implied that lacsRNA would resist coronin and get a long half-life during circulation [32]. Moreover, UV-Vis (Figure S1) and FT-IR (Figure 1E) spectroscopy further confirmed the successful assembly of lacsRNA as evidenced by characteristic absorbance given by Mercapto modified RNA. The valence states of $S$ and $A u$ in lacsRNA were explore by X-ray photoelectron spectroscopy (XPS) analysis, in which $S$ element presented +4 valence in line with the expected molecular composition in [RNA-S-Au$\left.{ }^{+}\right]_{n}$ (Figure 1F). Of note, the electronic energy signals given by Au in lacsRNA were low than the base electronic energy of Au atoms (Figure 1G), presumably because of the electron migration from $\mathrm{S}$ to $\mathrm{Au}$ after conjugation, thereby further proving the construction of the infinite Auric-sulfhydryl coordination polymeric miRNA. Collectively, these results demonstrated the successful conversion from miRNA to a near-spherical polymeric miRNA nanoparticle through a simple and mild "one-pot" chemistry.

\section{Physicochemical and pharmaceutical properties of lacsRNA}

As our design, lacsRNA should be endowed with the resistance against endonuclease, because of the steric hindrance of near-spherical nanostructure against enzyme recognition and binding [33, 34]. To explore it, nude miRNA and its corresponding lacsRNA were incubated in sterile PBS buffer containing $20 \%$ foetal bovine serum (FBS), and the residue RNA were semi-quantified by agarose gel electrophoresis (Figure $2 A$ ). In this case, lacsRNA obviously prolonged the half-time of RNA from $3.2 \pm 0.4 \mathrm{~h}$ to over 24 hours (Figure 2B)., indicating that lacsRNA strategy can overcome the poor systemic stability -the major 
pharmaceutical obstacle- of nuclear acid therapeutics. Next, to explore the cellular internalization of lacsRNA, Sulfo-Cyanine3 (Cy3) was 3'-terminally conjugated for the cellular uptakes examination by flow cytometry analysis. As shown in Figures $2 \mathrm{C}$ and D, lacsRNA (91.6\%) showed much more internalization into B16F10 melanoma cells than miRNA without transfection (39.4\%) after $6 \mathrm{~h}$ incubation. What's even more amazing is lacsRNA prevented the macrophages uptakes of miRNA (Figures $2 \mathrm{C}$ and $\mathrm{D}$ ), providing a highly favorable profile for circulation. This result compelled us to explore the colloidal stability of lacsRNA that is another important influencing factor for blood circulation. As expected, both in $\mathrm{pH} 7.4$ and $\mathrm{pH}$ 6.5, the incubation in PBS including $20 \%$ serum cannot alter the hydrodynamic diameter of lacsRNA (Figure 2E), indicative of low coronin and high colloidal stability. As a result, compared to miRNA alone, lacsRNA significantly improved the circulation time in blood after systematic injection quantified by the fluorescence signal from the labeled Cy3 fluorescein in miRNA (Figure 2F).

According to the enhanced permeability and retention (EPR) effect of nanoparticle, satisfactory circulation time always resulted in the promotional tumor accumulation. To verify it, we determined the biodistribution of lacsRNA in B16F10 homograft model of melanoma by inductively coupled plasma mass spectrometry (ICP-MS), which was used to quantify ${ }^{197} \mathrm{Au}$ concentrations in tissues. After intravenous injection of $2 \mathrm{mg} / \mathrm{Kg}$ lacsRNA, a time-dependent tendency for tumor accumulation can be found in the biodistribution results (Figure $1 \mathrm{G}$ ) and calculating the accumulation ratios of tumor versus normal organs (Figure $1 \mathrm{H}$ ). To visually examine the different tumor accumulation between miRNA and lacsRNA, Cy3-labaled samples were intravenously injected into the tumor-bearing mice. At $6 \mathrm{~h}$ postinjection, ex vivo fluorescence imaging and quantification (Figures $2 \mathrm{I}$ and $\mathrm{J}$ ) revealed over 4-times tumor accumulation in Cys-lacsRNA-treated mice in comparison to Cys-miRNA-treated mice. Taken together, these results demonstrated that lacsRNA strategy overcome the intrinsic weaknesses of nuclear acid therapeutic including poor systemic stability, rapid clearance, low membrane permeability and lack of tumor targeting.

\section{The safety evaluation of lacsRNA in vivo}

The superior performance of lacsRNA in pharmaceutical properties further compelled us to study its safety in vivo. To assess the safety of lacs-miR-30C, healthy C57/BL6 mice were intravenously injected with normal saline (Control) or lacs-miR-30C respectively every other day at a dosage of $10 \mathrm{mg} / \mathrm{kg}$, which was 5 -times the therapeutic dose ( $n=5$ per group). After 9 days of administration, no hepatotoxicity can be found as evidenced by the no difference of aspartate transaminase (ALT), alanine aminotransferase (AST) and pathological section of liver between Control and lacsRNA group (Figure 3A). Besides, the 9day administration have no effect on the blood urea nitrogen (BUN), creatinine (CREA) and Hematoxylin\&Eosin (H\&E) staining slices of kidney (Figure 3B), indicative of the hardly any nephrotoxicity. In addition, no hemolysis, myelosuppression, anemia, leukopenia and thrombopenia was found after lacs-miR-30C treatment (Figure 3C). Moreover, compared with control group, lacs-miR-30C treatment had almost no effect on body weight (Figure 3C, D), which further supported the highly favorable biosafety profile of lacs-miR-30C. Additionally, no pathological morphological changes can be 
found in the heart, lung, and spleen at lacs-miR-30C-treated mice. Collectively, these data illustrated that lacs-miR-30C is avirulent enough for clinical translation.

\section{lacsRNA Potently Suppressed Wnt/ß-catenin Pathway}

MiR-30c is known to inhibit oncogenic Wnt/ $\beta$-catenin activation through suppressing the expression of Bcl9 [21]. To comparatively investigate the potency of lacs-miR-30c and commercial miR-30c, B16F10 cell line- a kind of malignant and Wnt-hyperactive melanoma- was used to challenge their action via a 24hour incubation at the concentration of $50 \mathrm{nM}$. As expected, lacs-miR-30c enhanced the accumulation of miR-30C in cells and suppressed the expression of Bcl9 potently, whereas carrier-free miR-30C showed no difference between PBS-mock-treated control (Figures 4A and B). At a transcriptional level, lacs-miR-30C triggered 360 differentially expressed genes after 24-hour incubation on B16F10 cells as evidenced by RNA sequencing and subsequent clustering analysis $(n=3)$ (Figure $4 C)$. Gene set enrichment analysis (GSEA) exposed that suppression signatures were enriched in $\beta$-catenin gene sets in lacs-miR-30C-treated cells compared to the mock-treated cells (Figure 4D-F). Meanwhile, there was also a remarkably enrichment of suppression features presented in Wnt signaling pathway (Figure 4G-I). As a consequence, lacs-miR-30C induced the cell cycle arrest of cancer cells, as reflected in GSEA analysis in cell cycle, cell cycle checkpoints and cell cycle mitotic (Figures $4 \mathrm{~J}$ and S2). This resulted was proved again by the significantly decreased percentage of $S$ phage of cells measured by the PI-staining-derived cell cycle analysis (Figures $4 \mathrm{~K}$ and S3). Together, above results demonstrated that lacsRNA strategy resurrected the action of miR-30c and potently suppressed Wnt/ $\beta$-catenin pathway in vitro.

\section{lacsRNA potently suppressed Wnt/ $\beta$-catenin pathway in vivo and augmented the potency of chemotherapy}

To further test the in vivo potency of lacs-miR-30C, we comparatively explored its anti-cancer action with chemotherapeutic agent, and further investigated its synergistic sensitized effect with chemotherapy. Towards this end, a patient-derived-tumor-xenograft (PDX) model of colon carcinoma was established through subcutaneously transplanting surgically acquired colon tumor into the fossa iliaca of NOD/SCID mice (Figure $5 \mathrm{~A}$ ). In this model, the acquired colon tumor was characterized by identifying missense mutated oncogenes, including PIK3CA (S66T), APC (V1822D), EGFR (R521K), KRAS (G12D) (Figure 5B). Of note, V1822D mutation of APC suggested the hyper-action of Wnt signaling pathway, and G12D mutation of KRAS means the high malignancy and clinical incurability. When the tumor volume reached $100 \pm 30 \mathrm{~mm}^{3}$, tumor-bearing mice were treated with NS (Control), lacs-miR-30C, 5-FU (the first-line chemotherapy agent for colon cancer), or lacs-miR-30C/5-Fu combo every other day for 13 days, involving intravenous injections at the dosage of $2 \mathrm{mg} / \mathrm{kg}$ lacs-miR-30C, and/or of $5 \mathrm{mg} / \mathrm{kg}$ of 5 -FU (Figure 5A). At day 5, immunohistochemical staining image of tumors revealed that lacs-miR-30C remarkably down-regulated Bcl9 and effectively suppressed the $\beta$-catenin/Wnt signaling cascade as support by the decreased protein level of Bcl9, $\beta$-catenin, and two Wnt- downstream proteins, C-myc and Cyclin D (Figure 5C). As a result, compared with mock-treated group, lacs-miR-30C statistically 
significantly suppressed the tumor growth in more action than 5-Fu as evidence by the tumor volume curve (Figure 5D), tumor growth inhibition (TGI) effect (Figure 5E), tumor photos (Figure 5F) and tumor weights (Figure 5G). More importantly, the combination therapy between lacs-miR-30C and 5-Fu showed a significant increase anti-tumor action in contrast to the monotherapy of lacs-miR-30C or 5-Fu (Figures 5D-G), suggesting their synergistic sensitized effect. Moreover, proliferative cells analysis (ki67 staining) and apoptotic cells analysis (TUNEL staining) also supported these results (Figures $5 \mathrm{H}$ and I). Collectively, our data illustrated that lacs-miR-30C potently suppressed Wnt/ $\beta$-catenin pathway in vivo and augmented the anti-tumor action of the chemotherapeutic agent 5-FU.

\section{lacsRNA sensitized tumor immunotherapy}

Programmed cell death ligand 1 (PD-L1) on tumor cells or tumor derived exosomes binds programmed cell death 1 (PD-1) on T cells and efficiently stimulates cytotoxic $C D 8^{+} \mathrm{T}$ cell malfunctioning and apoptosis, allowing cancer cells to escape from the immune attack [17, 35, 36]. Anti PD-1 antibodies or anti PD-L1 antibodies are thus considered as effective anti-tumor drugs, but its anti-tumor response is always restrained to $T$ cell infiltration. In tumor, Wnt signaling results in T-cell exclusion and resistance to anti-PD-L1 or anti-PD-1 therapy [37]. Thus, we speculated that suppressing Wnt signaling cascade by lacs-miR-30C can sensitized the PD1/PD-L1 immuno-checkpoint blocking therapy. To verify it, we established an immunotherapeutic model in which C57/B6L mice were subcutaneously inoculated with B16F10 cells on the flank and treated with lacs-miR-30C, anti-PD-1 antibody or lacs-miR-30C/Anti-PD-1 antibody combo. As shown in Figures S4 and S5, lacs-miR-30C suppressed the $\beta$-catenin/Wnt signaling cascade in B16F10 tumor as support by the decreased protein level of Bcl9, $\beta$-catenin, C-myc and Cyclin D. Not surprisingly, compared with Anti-PD-1 or lacs-miR-30C monotherapy, the combo treatment resulted in a significantly decreased amounts of regulatory $T$ lymphocyte $\left(C D 4^{+} / C D 25^{+}\right.$cells, Figure. $\left.6 \mathrm{~A}, \mathrm{~B}\right)$ in sharp contrast to an increased number of tumor-infiltrating cytotoxic T lymphocyte (CD3 ${ }^{+} / \mathrm{CD}^{+}$cells, Figure. 6C, D). The inhibited tumor cell proliferation (Figure 6E) and the enhanced tumor cell apoptosis (Figure 6F) also supported the synergistic effect of lacs-miR-30C on anti-PD-1 therapy. As a result, lacsmiR-30C/Anti-PD-1 combo therapy led to dramatically increased TGI value ( $94.3 \% \mathrm{TGI})$ as compared to anti-PD-1 therapy (37.5\% TGI) or lacs-miR-30C therapy (62.7\% TGI) (Figures $6 \mathrm{G}$ and $\mathrm{H}$ ), in line with the results of the tumor photos (Figure 6I) and weights (Figure 6J). Collectively, these results provided abundant evidences that lacs-miR-30C treatment allowed overcoming T cell exclusion and amplified antitumor effects in anti-PD-1 antibody therapy through suppressing Wnt signaling cascade.

\section{Discussion}

Wnt signaling cascades play a crucial part in embryonic development, tissue homeostasis and stem cells proliferation and differentiation for all animals.[38] The aberrant activation of this signaling, however, underlies multiple human cancers, including but not limited to intestinal cancer, lung cancer, liver cancer and lymphoma.[39] In this case, dysregulated Wnt signaling cascades accumulates the transcriptional activator $\beta$-catenin in the cytoplasm and the nucleus, resulting in the promotion of the genes expression for malignant cell proliferation and metastasis. In the process, as a component of the aberrantly 
activated Wnt signaling pathway, $\beta$-catenin coactivators, including Bcl9, are always overexpressed in a variety of malignancies, and compose a stable complex with $\beta$-catenin to increase such malignant gene expression. [40] What's worse, dysregulated Wnt signaling cascades will reduce the infiltration of chemotherapeutics and T-lymphocytes (T-cell), resulting in the resistance of the chemotherapies and PD1/PD-L1 checkpoint-blockade Immunotherapies. [41-43] To addressed it, some researches have verified that the suppression of tumor-intrinsic active $\beta$-catenin signaling not only can inhibit the tumor progression, but also sensitized the chemotherapy and Immunotherapy ([44-47]). Of note, the present work amply proved these points: lacs-miR-30C effectively sensitized both potency of 5-Fu in PDX model of colon cancer (Figure 5) and Anti-PD1 in B16F10 homograft model of melanoma (Figure 6).

Although there has existed some success for Wnt/ $\beta$-catenin suppression, many still remain to be done, in especial of tumor specificity. The activated Wnt pathway is a common element in regulating stem cell/progenitor cell renewal and maintenance in noncancerous tissues and organs[48, 49]. As a results, Wnt/ $\beta$-catenin inhibitor always cause on-targeted toxicity, therefore none of them has yet been approved for clinical application [16]. To address this issue, Bcl9 that is highly expressed in tumors but not in the cells of tumor origin, has received considerable attention.[50] It has been reported that the oncogenic role of Bcl 9 can be rescued by siRNA/ShRNA-induced knockdown or the treatment with stapled Bcl9 peptide, both of which attenuated proliferation, metastasis, and resistance to therapy, highlighting the importance of Bcl9 for targeted oncotherapy [51, 52]. Thus, the development lacs-miR-30C possibly supplies the gap of safe and effective Wnt inhibitor for clinical transformation.

\section{Conclusion}

Herein, a general method through a mild and simple chemistry was established to convert therapeutic miRNA into a stable and bioavailable lacsRNA. Driven by aurophilicity, lacsRNA self-assembled into a spherical nanostructure with high colloid as well as anti-hydrolysis stability and low macrophage uptakes. As a consequence, lacsRNA presented the increased half-life period in circulation and accumulation at tumor sites in comparison to normal miRNA. More importantly, lacs-miR-30c showed no toxicity of viscera and sanguis system in the 5-time injection dosage of the treatment. Expectedly, lacsmiR-30c potently suppressed the Wnt signaling pathway in vitro and in vivo, and effectively sensitized both potency of 5-Fu in PDX model of colon cancer and Anti-PD1 in B16F10 homograft model of melanoma. In short, this work amply confirmed the design of lacsRNA as a general and viable strategy of nano-pharmaceutic to concert flimsy therapeutic miRNA into potential drugs. Considering from a broader perspective, the miRNA-initiated infinite coordination self-assembly strategy has distinct advantages in resurrecting nuclear acid therapeutics, probably bringing new inspiration to RNA-derived therapeutics of a great variety of human diseases including cancer.

\section{Declarations}

\section{Ethics approval and consent to participate}


All animal experiments in this work were performed in accordance with the guidelines of the Animal Ethics Committee of Xi'an Jiaotong University (approval number: 2020-276), Xi'an, China.

\section{Consent for publication}

Not applicable.

\section{Availability of data and materials}

The datasets used and/or analyzed during the current study are available from the corresponding author on reasonable request.

\section{Competing interests}

The authors declare no competing financial interest.

\section{Funding}

This work was supported by "The Young Talent Support Plan" of Xi'an Jiaotong University (For W. Liu and W. He), Thousand Talents Plan of Shaanxi Province (For W. He), National Natural Science Foundation of China (No. 81970915 for W. Liu, No. 22007076 for J. Yan and No. 32171256 for W. He), Natural Science Fund of Shaanxi for distinguished Young Scholars (2020JC33), Natural Science Basic Research Plan in Shaanxi Province of China (No. 2020JQ-092) and Key R \& D program of Shaanxi Province (No. 2019KW074 and No. 2021SF-033).

\section{Acknowledgements}

We thank Instrument Analysis Center of Xi'an Jiaotong University for their assistance with TEM, DLS, FTIR and XPS analysis. We also appreciate the help of RNA-seq analysis from BioNovoGene (Suzhou) Co., Ltd.

\section{Author information}

Liya Li and Wangxiao He contributed equally to this work

\section{Affiliations}

Institute for Stem Cell \& Regenerative Medicine, The Second Affiliated Hospital of Xi'an Jiaotong University, Xi'an 710004, China

Liya Li, Wangxiao He \& Wenjia Liu

Department of Medical Oncology and Department of Talent Highland, The First Affiliated Hospital of Xi'an Jiaotong University, Xi'an 710061, PR. China.

Wangxiao He 
National \& Local Joint Engineering Research Center of Biodiagnosis and Biotherapy, The Second Affiliated Hospital of Xi'an Jiaotong University, Xi'an, 710004, PR. China.

Weiming You \& Jin Yan

\section{Contributions}

WH, JY and WL supervised and conceptualized the study, LL and WH performed most of the experiments, $\mathrm{WH}, \mathrm{LL}$ and WL wrote the manuscript, WY helped with project design and provided guidance on some experiments. All authors read and approved the final manuscript.

\section{Corresponding authors}

Correspondence to Wangxiao He, Jin Yan or Wenjia Liu.

\section{References}

1. Ganju A, Khan S, Hafeez BB, Behrman SW, Yallapu MM, Chauhan SC, Jaggi M: miRNA nanotherapeutics for cancer. Drug Discov Today 2017, 22:424-432.

2. Zhao XH, Wang P, Liu J, Zheng J, Liu YH, Chen JJ, Xue YX: Gas5 Exerts Tumor-suppressive Functions in Human Glioma Cells by Targeting miR-222. Molecular Therapy 2015, 23:1899-1911.

3. Wen SY, Lin Y, Yu YQ, Cao SJ, Zhang R, Yang XM, Li J, Zhang YL, Wang YH, Ma MZ, et al: miR-506 acts as a tumor suppressor by directly targeting the hedgehog pathway transcription factor Gli3 in human cervical cancer. Oncogene 2015, 34:717-725.

4. Kai ZS, Pasquinelli AE: MicroRNA assassins: factors that regulate the disappearance of miRNAs. Nature Structural \& Molecular Biology 2010, 17:5-10.

5. Yin H, Kanasty RL, Eltoukhy AA, Vegas AJ, Dorkin JR, Anderson DG: Non-viral vectors for gene-based therapy. Nature Reviews Genetics 2014, 15:541-555.

6. Morille M, Passirani C, Vonarbourg A, Clavreul A, Benoit JP: Progress in developing cationic vectors for non-viral systemic gene therapy against cancer. Biomaterials 2008, 29:3477-3496.

7. Pattni BS, Chupin VV, Torchilin VP: New Developments in Liposomal Drug Delivery. Chemical Reviews 2015, 115:10938-10966.

8. Bozzuto G, Molinari A: Liposomes as nanomedical devices. International Journal of Nanomedicine 2015, 10:975-999.

9. Huang SN, Duan SF, Wang J, Bao SJ, Qiu XJ, Li CM, Liu Y, Yan LJ, Zhang ZZ, Hu YR: Folic-AcidMediated Functionalized Gold Nanocages for Targeted Delivery of Anti-miR-181b in Combination of Gene Therapy and Photothermal Therapy against Hepatocellular Carcinoma. Advanced Functional Materials 2016, 26:2532-2544.

10. Chen X, Gu S, Chen BF, Shen WL, Yin Z, Xu GW, Hu JJ, Zhu T, Li G, Wan C, et al: Nanoparticle delivery of stable miR-199a-5p agomir improves the osteogenesis of human mesenchymal stem cells via the 
HIF1a pathway. Biomaterials 2015, 53:239-250.

11. Yu M, Lei B, Gao CB, Yan J, Ma PX: Optimizing surfaceengineered ultra-small gold nanoparticles for highly efficient miRNA delivery to enhance osteogenic differentiation of bone mesenchymal stromal cells. Nano Research 2017, 10:49-63.

12. Yu M, Xue YM, Ma PX, Mao C, Lei B: Intrinsic Ultrahigh Drug/miRNA Loading Capacity of Biodegradable Bioactive Glass Nanoparticles toward Highly Efficient Pharmaceutical Delivery. Acs Applied Materials \& Interfaces 2017, 9:8460-8470.

13. Yu M, Yan J, He W, Li C, Ma PX, Lei B: Synthetic $\theta$-Defensin Antibacterial Peptide as a Highly Efficient Nonviral Vector for Redox-Responsive miRNA Delivery. Adv Biosyst 2017, 1:1700001.

14. Li HJ, Du JZ, Du XJ, Xu CF, Sun CY, Wang HX, Cao ZT, Yang XZ, Zhu YH, Nie SM, Wang J: Stimuliresponsive clustered nanoparticles for improved tumor penetration and therapeutic efficacy. Proceedings of the National Academy of Sciences of the United States of America 2016, 113:41644169.

15. Zanganeh S, Hutter G, Spitler R, Lenkov O, Mahmoudi M, Shaw A, Pajarinen JS, Nejadnik H, Goodman $\mathrm{S}$, Moseley $\mathrm{M}$, et al: Iron oxide nanoparticles inhibit tumour growth by inducing pro-inflammatory macrophage polarization in tumour tissues. Nature Nanotechnology 2016, 11:986-994.

16. He W, Yan J, Jiang W, Li S, Qu Y, Niu F, Yan Y, Sui F, Wang S, Zhou Y: Peptide-Induced Self-Assembly of Therapeutics into a Well-Defined Nanoshell with Tumor-Triggered Shape and Charge Switch. Chem Mater 2018, 30:7034-7046.

17. Yan J, He W, Li X, You W, Liu X, Lin S, Chen J, Zhao Y, Zhang Y, Ji F: Carnosic acid-induced co-selfassembly of metal-peptide complexes into a nanocluster-based framework with tumor-specific accumulation for augmented immunotherapy. Chem Eng J 2021, 416:129141.

18. Zheng X, Yan J, You W, Li F, Diao J, He W, Yao Y: De Novo Nano-Erythrocyte Structurally Braced by Biomimetic Au(I)-peptide Skeleton for MDM2/MDMX Predation toward Augmented Pulmonary Adenocarcinoma Immunotherapy. Small 2021:e2100394.

19. He W, Yan J, Li Y, Yan S, Wang S, Hou P, Lu W: Resurrecting a p53 peptide activator - An enabling nanoengineering strategy for peptide therapeutics. J Control Release 2020, 325:293-303.

20. Yan J, Yao Y, Yan S, Gao R, Lu W, He W: Chiral Protein Supraparticles for Tumor Suppression and Synergistic Immunotherapy: An Enabling Strategy for Bioactive Supramolecular Chirality Construction. Nano Lett 2020, 20:5844-5852.

21. She J, Li Y, Yan S, Yan Y, Liu D, Li S, Guo Y, Xue Y, Yao Y, Yan J, He W: De novo supraparticle construction by a self-assembled Janus cyclopeptide to tame hydrophilic microRNA and hydrophobic molecule for anti-tumor cocktail therapy and augmented immunity. Chem Eng J 2020, 401:126080.

22. Barenholz Y: Doxil(R)-the first FDA-approved nano-drug: lessons learned. J Control Release 2012, 160:117-134.

23. Jain RK, Stylianopoulos T: Delivering nanomedicine to solid tumors. Nat Rev Clin Oncol 2010, 7:653664. 
24. Mei H, Cai S, Huang D, Gao H, Cao J, He B: Carrier-free nanodrugs with efficient drug delivery and release for cancer therapy: From intrinsic physicochemical properties to external modification. Bioactive Materials 2022, 8:220-240.

25. Karaosmanoglu S, Zhou M, Shi B, Zhang X, Williams GR, Chen X: Carrier-free nanodrugs for safe and effective cancer treatment. J Control Release 2021, 329:805-832.

26. Imaz I, Rubio-Martinez M, Garcia-Fernandez L, Garcia F, Ruiz-Molina D, Hernando J, Puntes V, Maspoch D: Coordination polymer particles as potential drug delivery systems. Chem Commun 2010, 46:4737-4739.

27. Luo S, Wang Y, Shen S, Tang P, Liu Z, Zhang S, Wu D: IR780-Loaded Hyaluronic Acid@GossypolFe(III)-EGCG Infinite Coordination Polymer Nanoparticles for Highly Efficient Tumor Photothermal/Coordinated Dual Drugs Synergistic Therapy. Adv Funct Mater 2021, 31:2100954.

28. Zhao JJ, Lin JH, Zhu D, Wang XJ, Brooks D, Chen M, Chu ZB, Takada K, Ciccarelli B, Admin S, et al: miR-30-5p Functions as a Tumor Suppressor and Novel Therapeutic Tool by Targeting the Oncogenic Wnt/beta-Catenin/BCL9 Pathway. Cancer Research 2014, 74:1801-1813.

29. Negishi Y, Nobusada K, Tsukuda T: Glutathione-protected gold clusters revisited: Bridging the gap between gold (I)- thiolate complexes and thiolate-protected gold nanocrystals. J Am Chem Soc 2005, 127:5261-5270.

30. Yan J, Ji F, Yan S, You W, He W: A general-purpose Nanohybrid fabricated by Polymeric Au(I)-peptide precursor to wake the function of Peptide Therapeutics. Theranostics 2020, 10:8513-8527.

31. He W, Wang S, Yan J, Qu Y, Jin L, Sui F, Li Y, You W, Yang G, Yang Q, et al: Self-Assembly of Therapeutic Peptide into Stimuli-Responsive Clustered Nanohybrids for Cancer-Targeted Therapy. Adv Funct Mater 2019, 29:1807736.

32. He W, Yan J, Wang L, Lei B, Hou P, Lu W, Ma PX: A lanthanide-peptide-derived bacterium-like nanotheranostic with high tumor-targeting, -imaging and -killing properties. Biomaterials 2019, 206:13-24.

33. Yan J, He W, Yan S, Niu F, Liu T, Ma B, Shao Y, Yan Y, Yang G, Lu W, et al: Self-Assembled PeptideLanthanide Nanoclusters for Safe Tumor Therapy: Overcoming and Utilizing Biological Barriers to Peptide Drug Delivery. ACS Nano 2018, 12:2017-2026.

34. Yan J, Yan S, Hou P, Lu W, Ma PX, He W, Lei B: A Hierarchical Peptide-Lanthanide Framework To Accurately Redress Intracellular Carcinogenic Protein-Protein Interaction. Nano Lett 2019, 19:79187926.

35. Wang Y, Zhou S, Yang F, Qi X, Wang X, Guan X, Shen C, Duma N, Vera Aguilera J, Chintakuntlawar A, et al: Treatment-Related Adverse Events of PD-1 and PD-L1 Inhibitors in Clinical Trials: A Systematic Review and Meta-analysis. JAMA Onco/ 2019, 5:1008-1019.

36. Liu J, Yan J, Yan S, Wang Y, Zhang R, Hou P, He W, Ji M: Biomimetic and Self-Assembled Nanoclusters Targeting $\beta$-Catenin for Potent Anticancer Therapy and Enhanced Immunotherapy. Nano Lett 2019, 19:8708-8715. 
37. Feng M, Jin JQ, Xia L, Xiao T, Mei S, Wang X, Huang X, Chen J, Liu M, Chen C, et al: Pharmacological inhibition of $\beta$-catenin/BCL9 interaction overcomes resistance to immune checkpoint blockades by modulating Treg cells. Sci Adv 2019, 5:eaau5240.

38. Moon RT, Kohn AD, De Ferrari GV, Kaykas A: WNT and beta-catenin signalling: diseases and therapies. Nat Rev Genet 2004, 5:691-701.

39. Reya T, Clevers H: Wnt signalling in stem cells and cancer. Nature 2005, 434:843-850.

40. Ling XH, Chen ZY, Luo HW, Liu ZZ, Liang YK, Chen GX, Jiang FN, Zhong WD: BCL9, a coactivator for Wnt/-catenin transcription, is targeted by miR-30c and is associated with prostate cancer progression. Oncology Letters 2016, 11:2001-2008.

41. Lake RA, Robinson BW: Immunotherapy and chemotherapy-a practical partnership. Nat Rev Cancer 2005, 5:397-405.

42. Chen M, Zhou X, Chen R, Wang J, Ye RD, Wang Y, Wu C, Mahato RI: Nano-carriers for delivery and targeting of active ingredients of Chinese medicine for hepatocellular carcinoma therapy. Materials Today 2019, 25:66-87.

43. Spranger S, Bao R, Gajewski T: Melanoma-intrinsic $\beta$-catenin signaling prevents $T$ cell infiltration and anti-tumor immunity. Journal for ImmunoTherapy of Cancer 2014, 2:015.

44. Martins-Neves SR, Paiva-Oliveira DI, Wijers-Koster PM, Abrunhosa AJ, Fontes-Ribeiro C, Bovee JVMG, Cleton-Jansen AM, Gomes CMF: Chemotherapy induces sternness in osteosarcoma cells through activation of Wnt/beta-catenin signaling. Cancer Letters 2016, 370:286-295.

45. Wang YX, Liu CZ, Luo M, Zhang ZY, Gong JN, Li JJ, You L, Dong L, Su R, Lin HS, et al: ChemotherapyInduced miRNA-29c/Catenin-delta Signaling Suppresses Metastasis in Gastric Cancer. Cancer Research 2015, 75:1332-1344.

46. Gattinoni L, Ji Y, Restifo NP: Wnt/beta-Catenin Signaling in T-Cell Immunity and Cancer Immunotherapy. Clinical Cancer Research 2010, 16:4695-4701.

47. Suryawanshi A, Tadagavadi RK, Swafford D, Manicassamy S: Modulation of inflammatory Responses by wnt/beta-Catenin Signaling in Dendritic Cells: A Novel immunotherapy Target for Autoimmunity and Cancer. Frontiers in Immunology 2016, 7.

48. Merrill BJ: Wnt Pathway Regulation of Embryonic Stem Cell Self-Renewal. Cold Spring Harbor Perspectives in Biology 2012, 4.

49. Xiao L, Yuan X, Sharkis SJ: Activin A maintains self-renewal and regulates fibroblast growth factor, Wnt, and bone morphogenic protein pathways in human embryonic stem cells. Stem Cells 2006, 24:1476-1486.

50. Takada K, Zhu D, Bird GH, Sukhdeo K, Zhao JJ, Mani M, Lemieux M, Carrasco DE, Ryan J, Horst D, et al: Targeted Disruption of the BCL9/beta-Catenin Complex Inhibits Oncogenic Wnt Signaling. Science Translational Medicine 2012, 4.

51. Deka J, Wiedemann N, Anderle P, Murphy-Seiler F, Bultinck J, Eyckerman S, Stehle JC, Andre S, Vilain $\mathrm{N}$, Zilian O, et al: Bcl9/Bcl9l Are Critical for Wnt-Mediated Regulation of Stem Cell Traits in Colon Epithelium and Adenocarcinomas. Cancer Research 2010, 70:6619-6628. 

Early Stages of Intestinal Tumor Progression. Gastroenterology 2011, 141:1359-U1810.

\section{Figures}

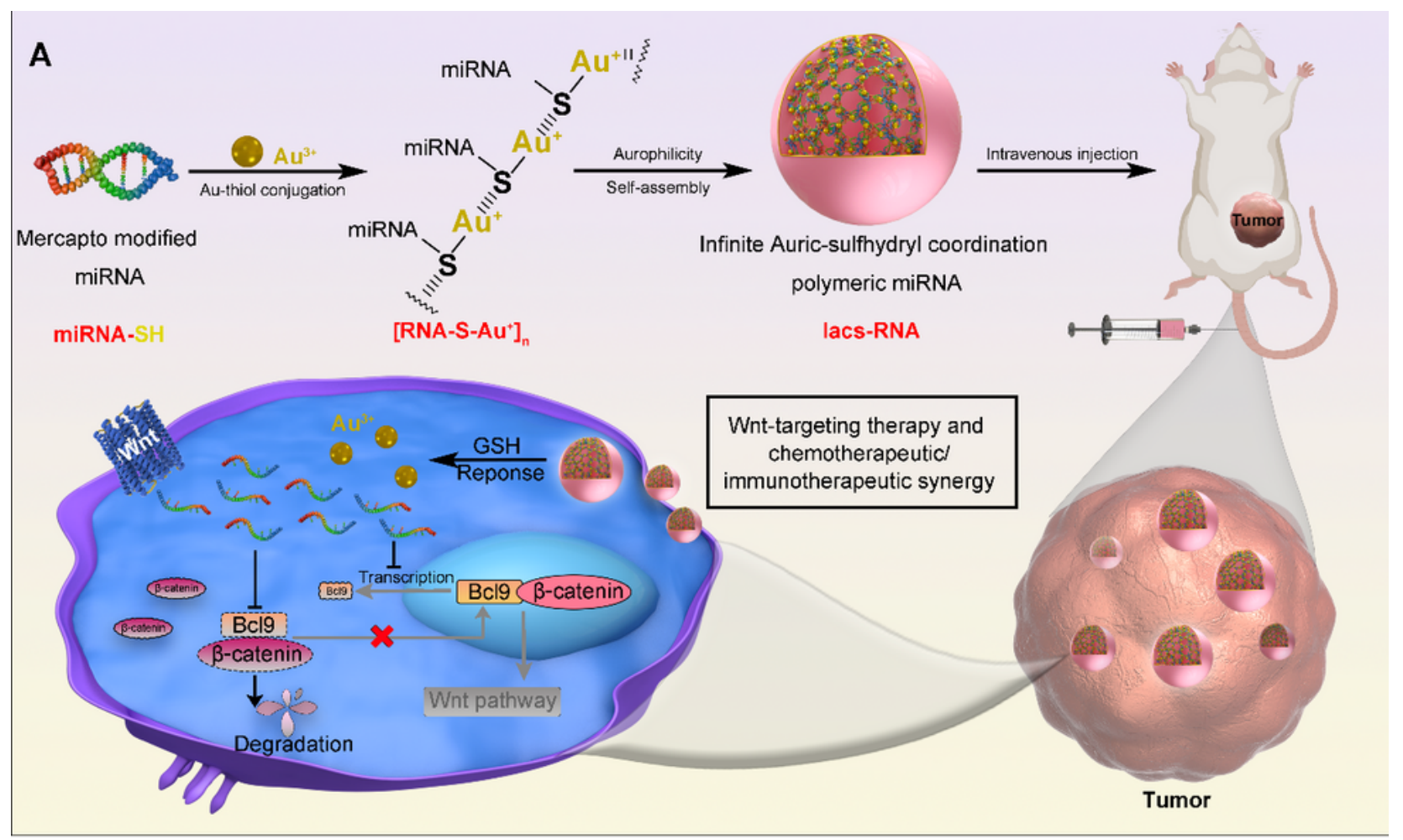

B

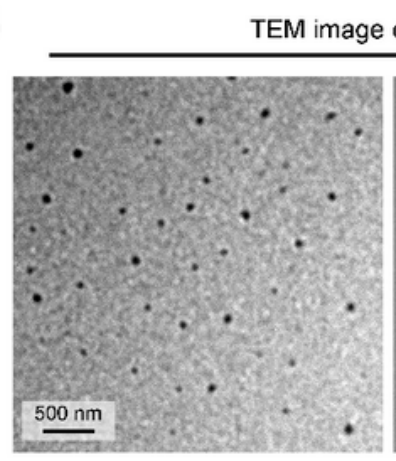

C

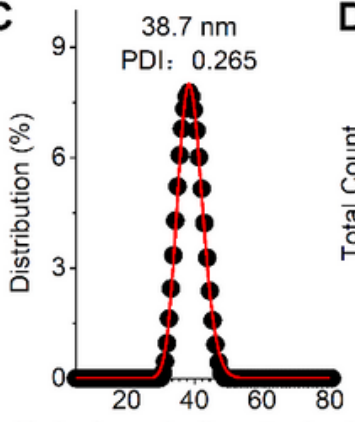

Hydrodynamic diameter $(\mathrm{nm})$

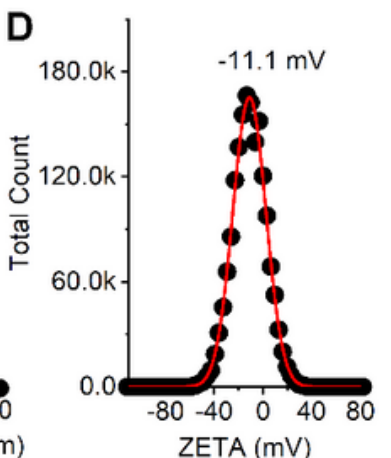

ZETA $(\mathrm{mV})$
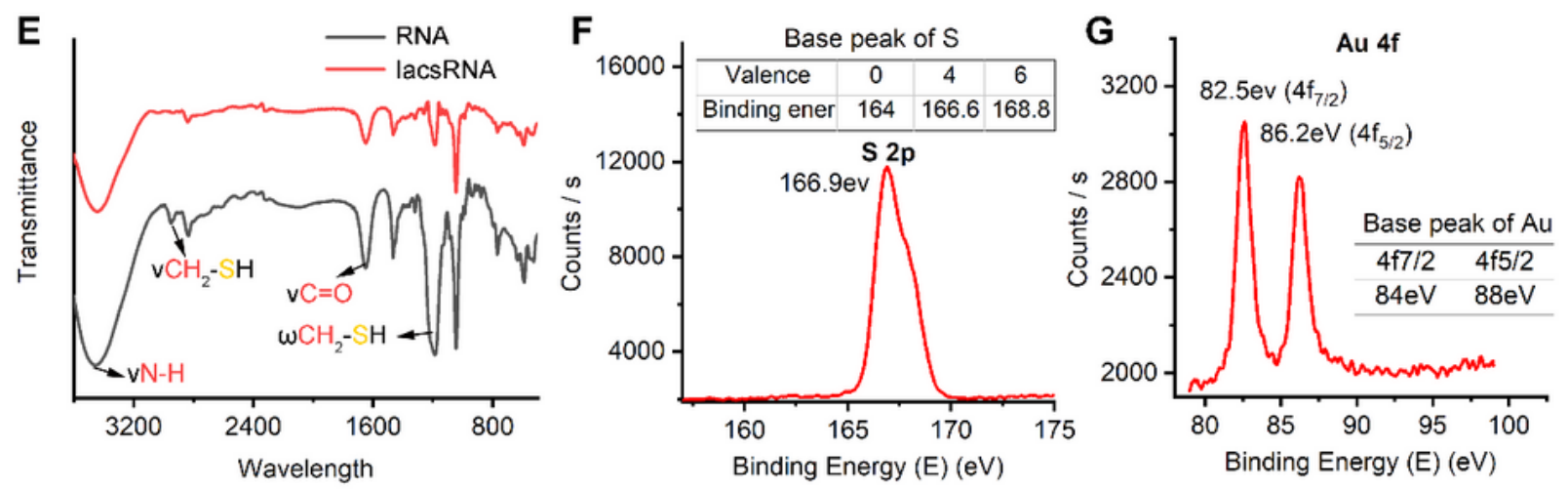

Figure 1 
Fabrication and therapeutic mechanism of lascRNA. (A) Illustration of the synthesis and tumor response procedure of lacsRNA. (B) TEM images of lacsRNA. (C and D) Hydrodynamic diameter (C) and ZETA potential (D) of lacsRNA measured in PBS buffer at pH 7.4. (E) FT-IR spectra of lacsRNA and RNA. The band at $1200 \mathrm{~cm}-1$ which was attributed to the stretching vibration of $-\mathrm{SH}$. (F and $\mathrm{G}$ ) S 2p XPS spectra (F) and $A u 4 f$ XPS spectra $(G)$ of lacsRNA.

A

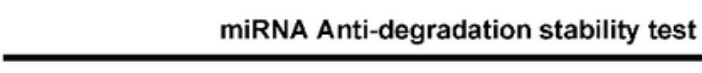

Incubation in PBS containing 20\% FBS

$C$ : nude miRNA $E$ : lacsRNA

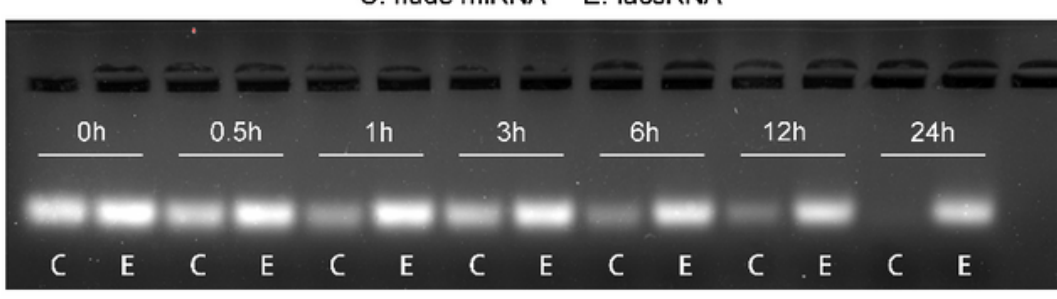

C
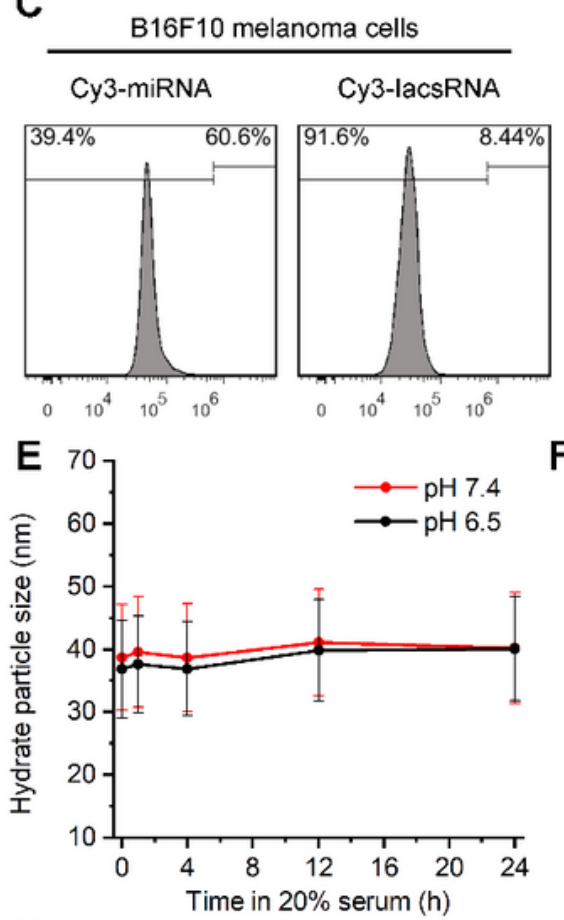

H

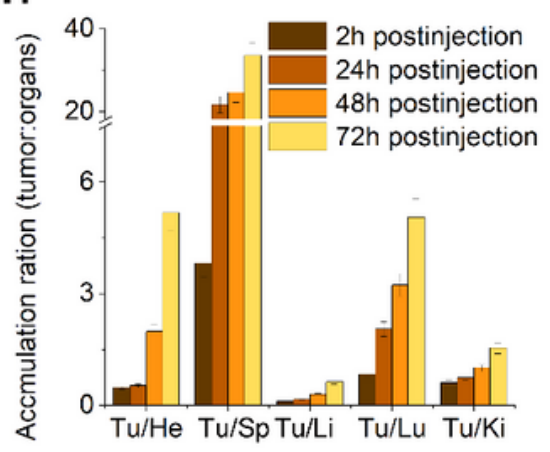

Cy3-lacsRNA
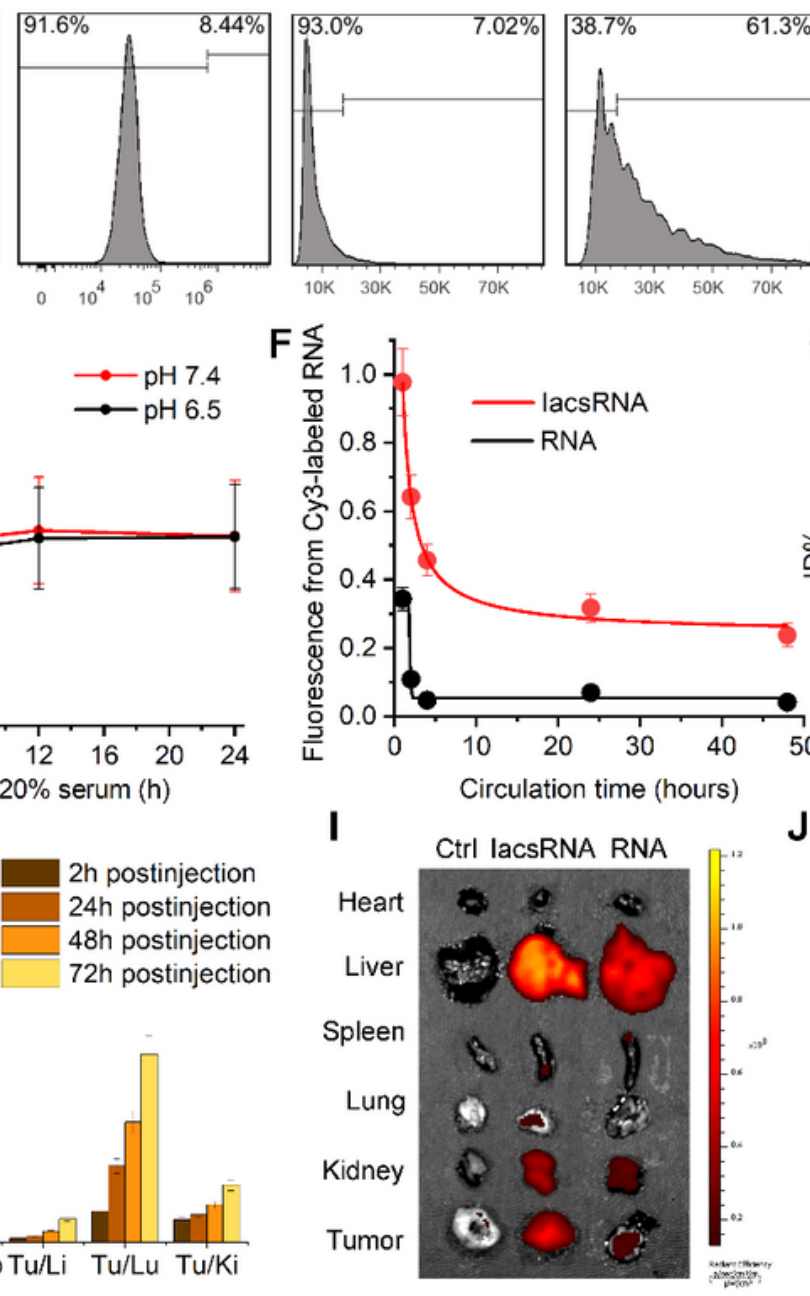

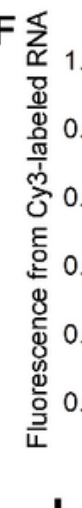

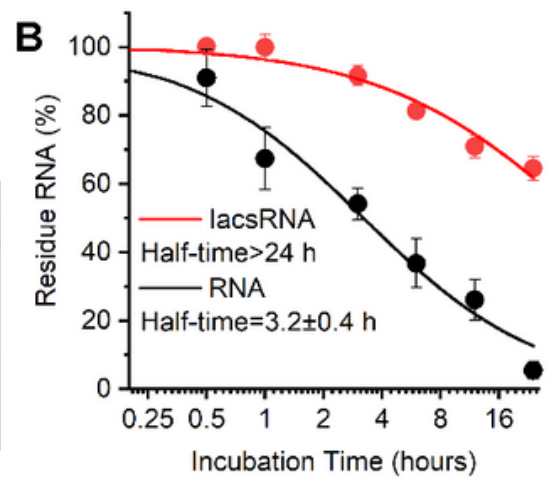

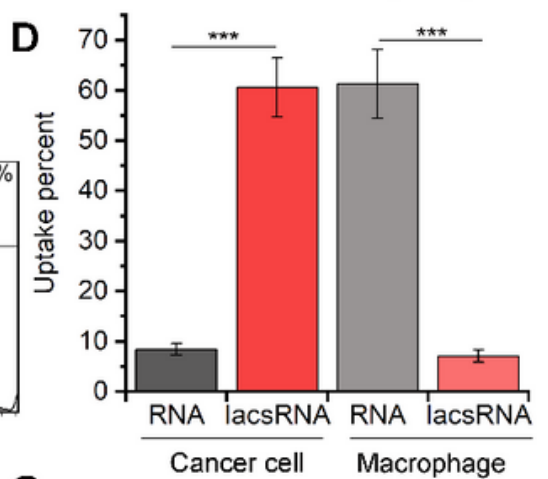

G
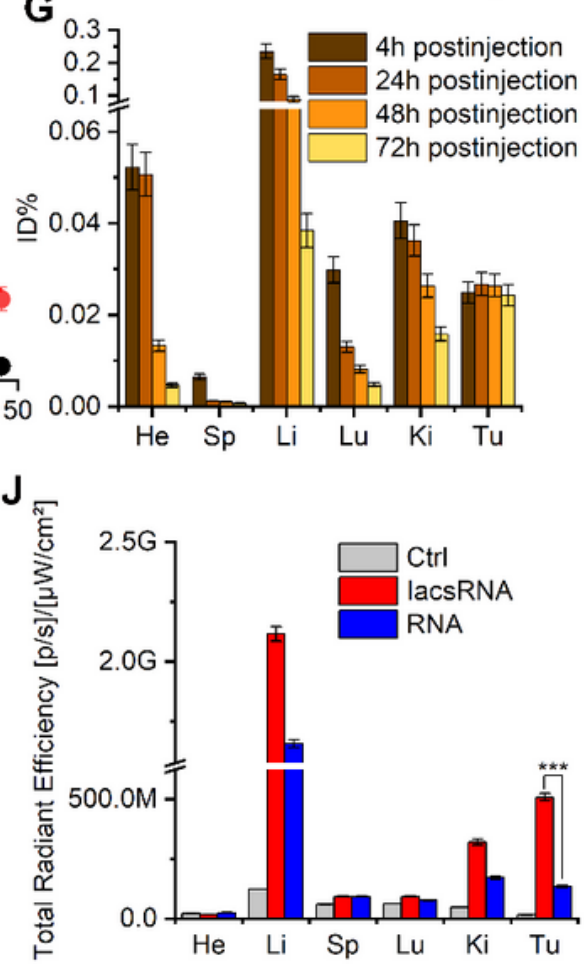

Figure 2 
Physicochemical and pharmaceutical properties of lacsRNA. (A and B) Anti-degradation stability test (A) and quantitative analysis (B) of lacsRNA and nude miRNA by gel electrophoresis. ( $C$ and D) Flow cytometry analysis (C) and quantification (D) of different cells uptake of $1 \mu \mathrm{M}$ Cy3-labelled miRNA and lacsRNA after $6 \mathrm{~h}$ incubations. (E) Colloidal stability of lacsRNA suspending in PBS containing $20 \% \mathrm{FBS}$ at pH7.4 and 6.5 measured by DLS. (F) Blood-circulation curves of lacsRNA and RNA in healthy C57/B6 mice quantified by the fluorescence signal from the labeled Cy3 fluorescein in miRNA. (G) organ distribution of lacsRNA in BALB/c mice after systemic injection. Serial sacrifices were carried out at $2 \mathrm{~h}$, $24 \mathrm{~h}, 48 \mathrm{~h}$ and $72 \mathrm{~h}$ after injection. Several organs/tissues, including heart, liver, spleen, lung, kidney and tumor were isolated to determine gold concentrations by ICP-MS. He, heart, Sp, spleen, Li, liver, Lu, lung, $\mathrm{Ki}$, kidney, Tu, tumor. $(\mathrm{H})$ tumor-to-organ ratios for lacsRNA at $2 \mathrm{~h}, 24 \mathrm{~h}, 48 \mathrm{~h}$ and $72 \mathrm{~h}$ after injection. (I and $\mathrm{J})$ Tissue distribution (I) and quantification $(\mathrm{J})$ of lacsRNA. The fluorescence signal from the tumors and normal organ after lacsRNA intraperitoneal injection $(200 \mu \mathrm{L}, 1 \mathrm{mM} \mathrm{Au})$ at $1 \mathrm{~h}$. The data were presented as mean \pm s.d. ${ }^{*}, p<0.05, * *, p<0.01, * * *, p<0.001$. 


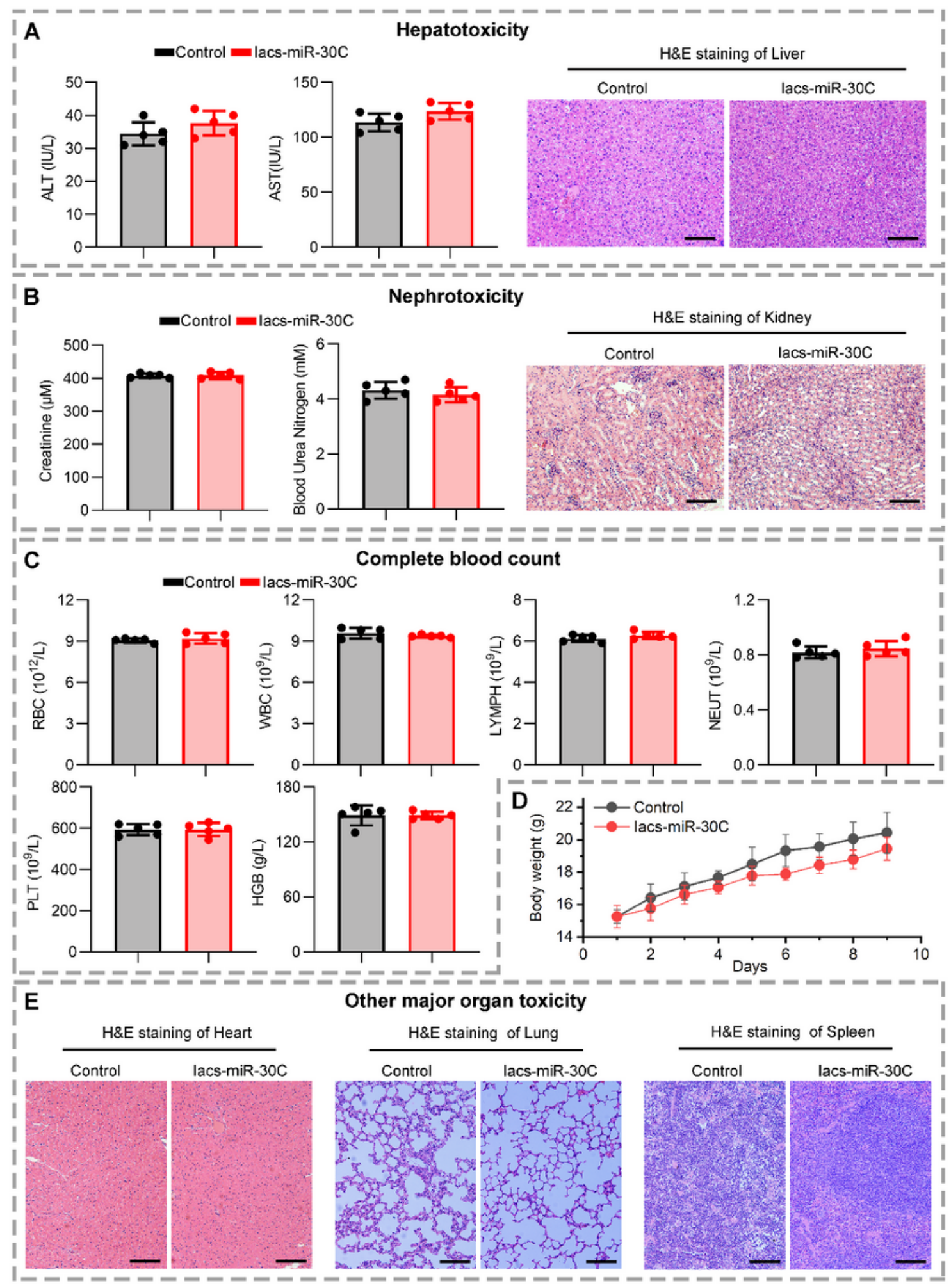

Figure 3

The biosafety evaluation of lacsRNA in vivo. (A) Hepatotoxicity testing of the lacsRNA measured by aspartate transaminase (ALT), alanine aminotransferase (AST), and pathological section of liver (scale bar: $100 \mu \mathrm{m})$. (B) Nephrotoxicity testing of the lacsRNA measured by blood urea nitrogen (BUN), creatinine

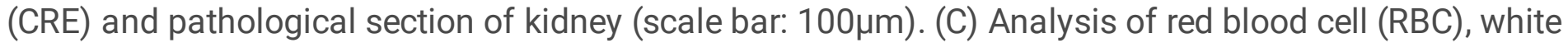
blood cell (WBC), lymphocyte (LYMPH), Neutrophil (NEUT), Platelets (PLT) and Hemoglobin (HGB) in mice 
blood with the indicated treatments. (D) Body weight of mice with the indicated treatments. (E) Toxicity testing of the lacsRNA measured by pathological section of heart, lung and spleen. The data were presented as mean \pm s.d. *, $p<0.05$.

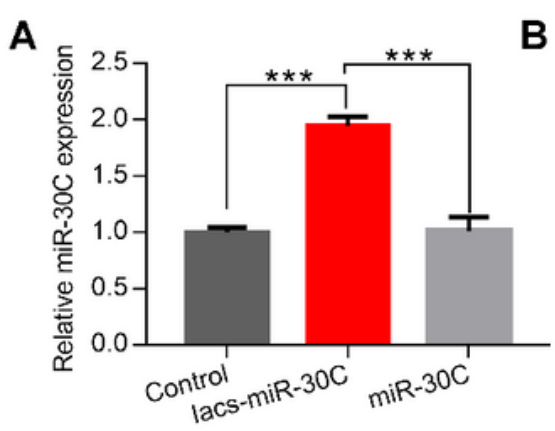

D

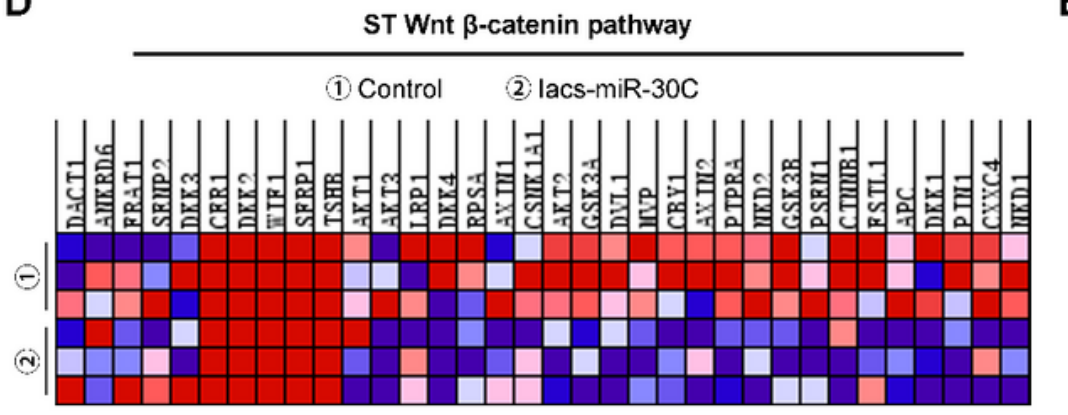

$\mathrm{F}$

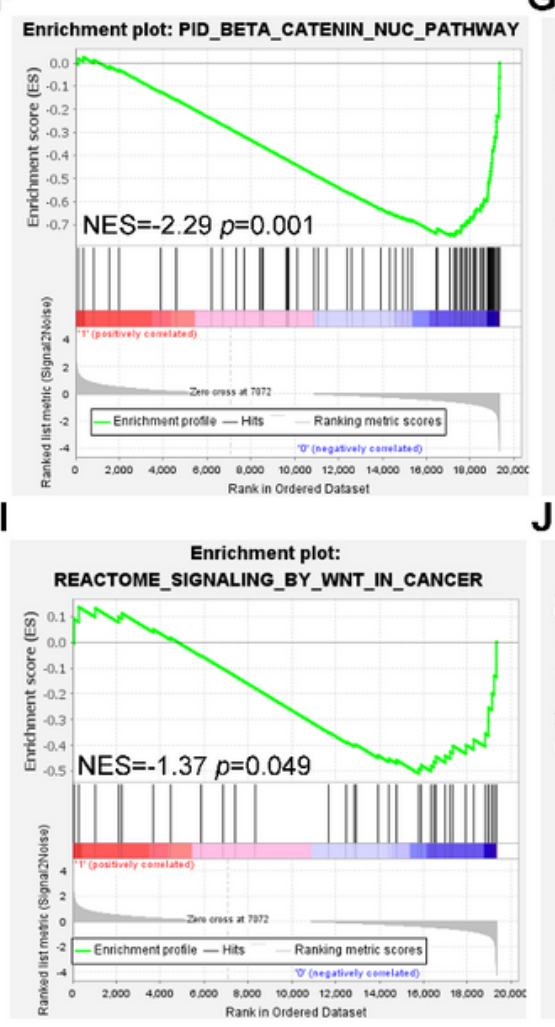

B

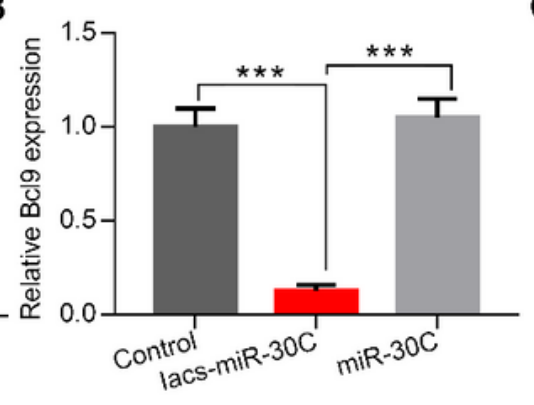

C

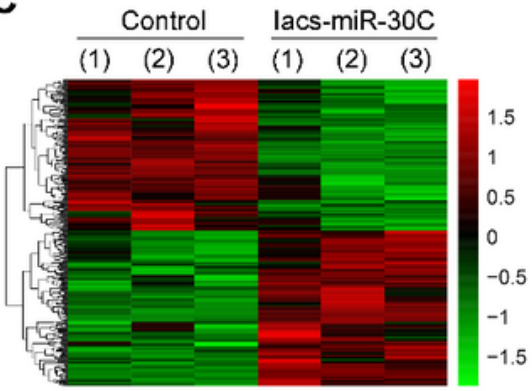

E

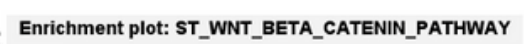

西

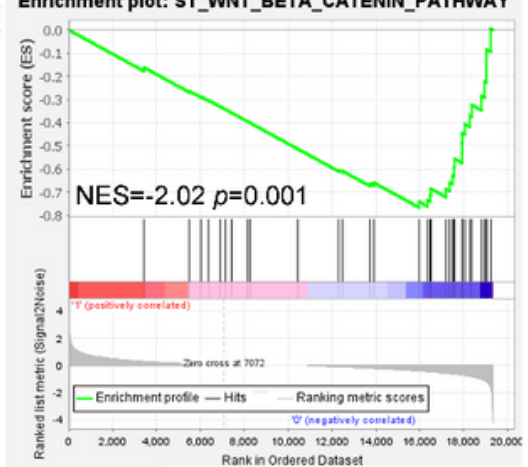

$\mathbf{H}$

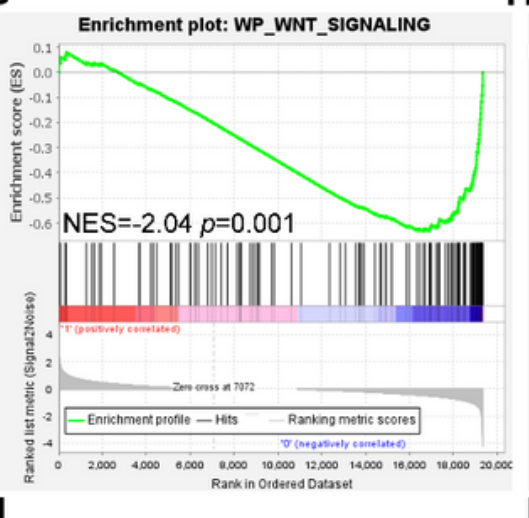

Enrichment plot:
REACTOME_BETA_CATENIN_INDEPENDENT_WNT_SIGN

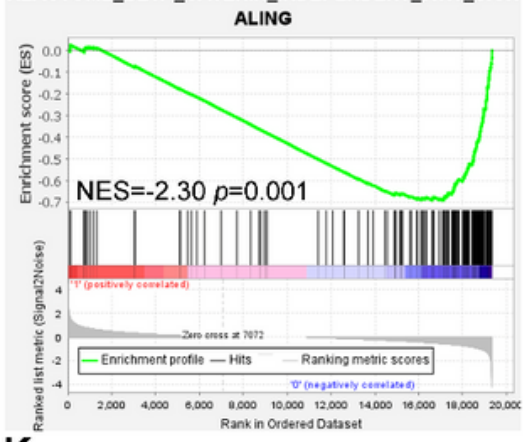

K

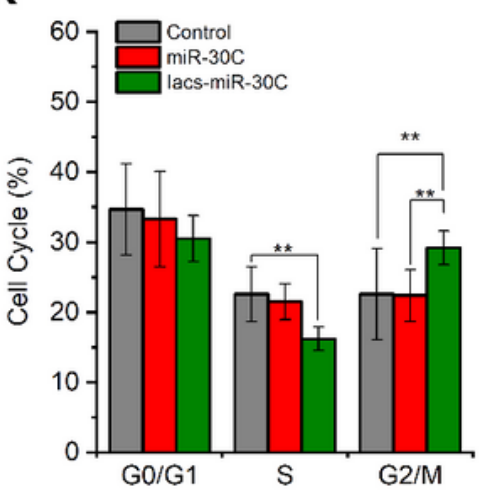

Figure 4

lacsRNA targeting Bcl9 inhibits Wnt pathway. (A and B) B16F10 cells were treated with lacsRNA and miR$30 \mathrm{C}$ for $24 \mathrm{~h}$ at the dosage of $50 \mathrm{nM}$, and qRT-PCR was performed to analyze the expressions of miR-30C 
(A) and Bcl9 (B). (C) Hierarchical clustering of genes differentially expressed in B16F10 cells after exposure to 50nM lacsRNA for $24 \mathrm{~h}$ compared with miR-30C-treated cells $(n=3)$. (D) Hierarchical clustering of genes in Wnt/ $\beta$-catenin signaling pathway. (E-J) Gene set enrichment analysis (GSEA) showing the Wnt/ $\beta$-catenin pathway, $\beta$-catenin signaling pathway and cell cycle differentially expressed in response to lacsRNA. KEGG, Kyoto Encyclopedia of Genes and Genomes, PID, Pathway Interaction Database, Nes, normalized enrichment score. (K) Cell cycle was analyzed by flow cytometry. The data were presented as mean \pm s.d. ${ }^{*}, p<0.05, * *, p<0.01$.

A

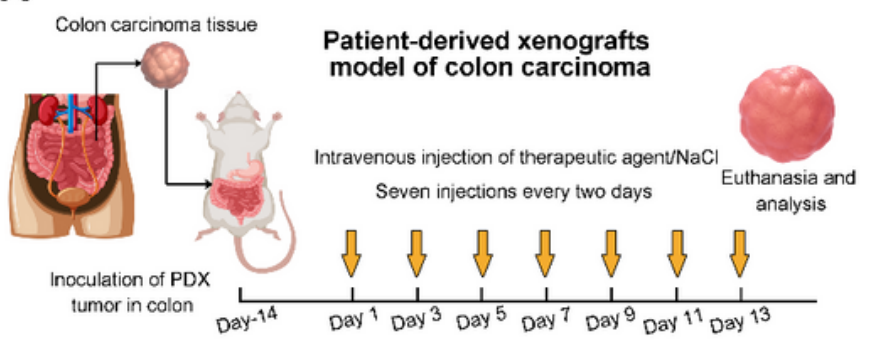

B

\section{Exon sequencing of key genes}

\begin{tabular}{cc|cc}
\hline Gene & Mutations & Gene & Mutations \\
\hline TP53 & WT & PIK3CA & S66T \\
TP73 & WT & APC & V1822D \\
PTEN & WT & EGFR & R521K \\
NRAS & WT & AKT1 & WT \\
BRAF & WT & KRAS & G12D \\
\hline
\end{tabular}

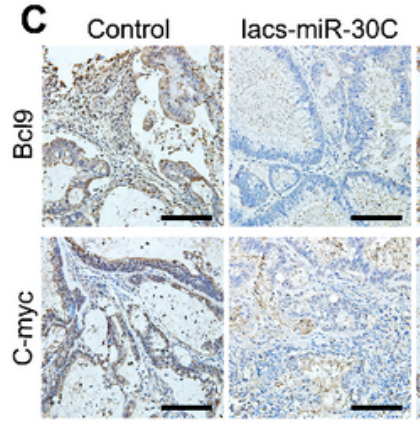

5-FU lacs-miR-30c/5-FU

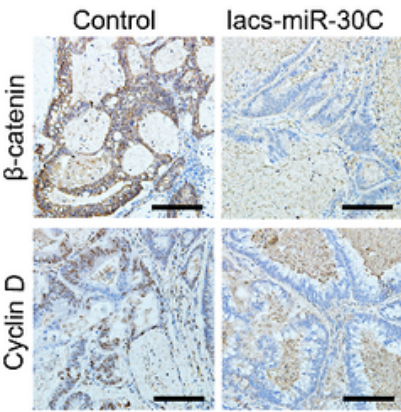

5-FU lacs-miR-30C/5-FU
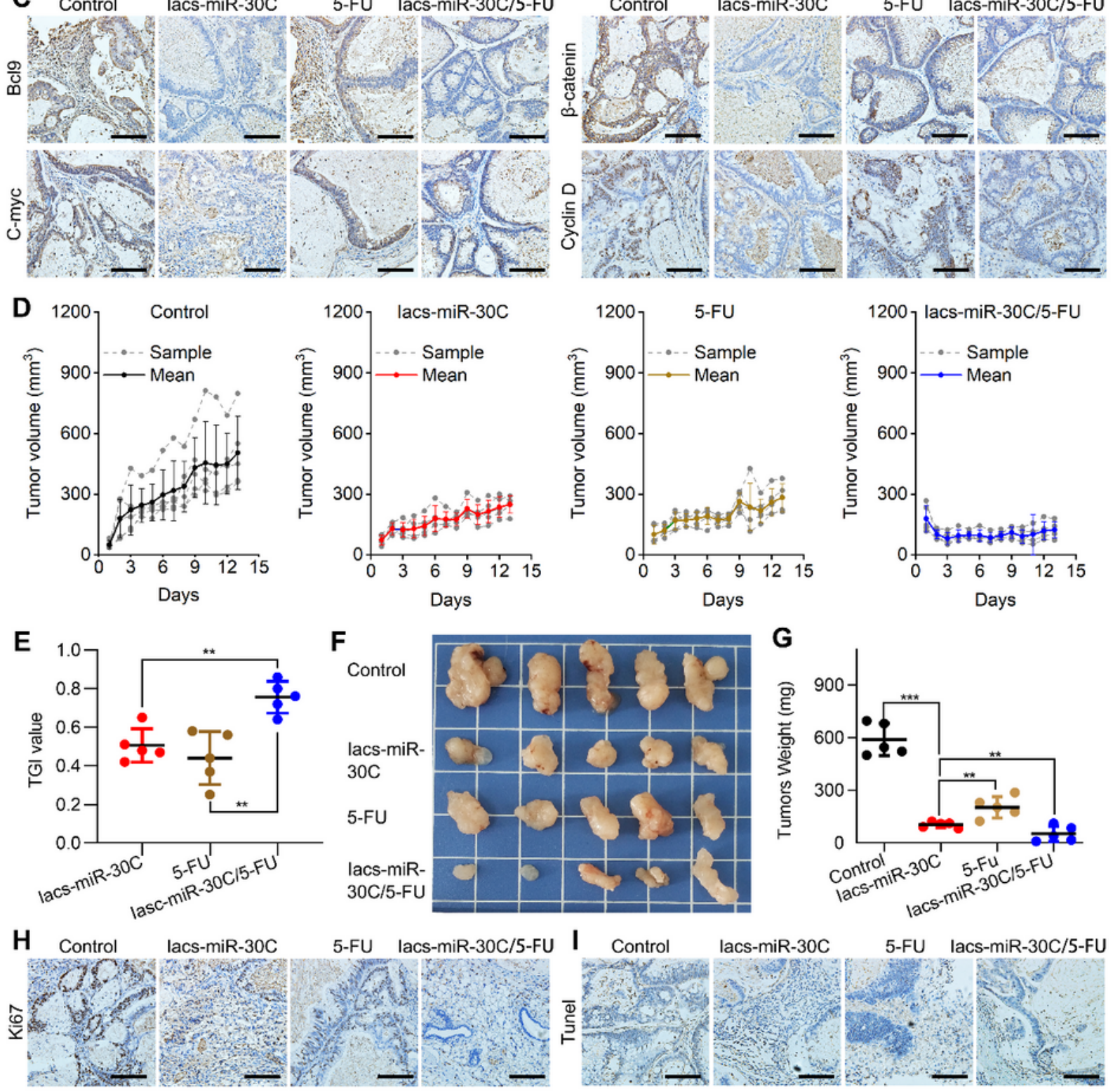


\section{Figure 5}

lacsRNA in vivo inhibited patient-derived tumor xenograft colon carcinoma in NOD/SCID mice. (A) Schematic diagram of PDX model construction. (B) Detection of tumor mutations by exon sequencing. WT stands for wild type. (C) Representative tumor sections after the indicated treatment staining by immunohistochemistry (scale bar: $100 \mu \mathrm{m}$ ). (D) Tumor growth curves in PDX mouse model mice with the indicated treatments $(n=5)$. (E) Tumor growth inhibition $(T G I)$ with the indicated treatment at day 13. ( $F$ and $\mathrm{G}$ ) Representative photographs $(\mathrm{F})$ and weight of tumor $(\mathrm{G})$ after the indicated treatments. ( $\mathrm{H}$ and $\mathrm{I})$ Ki67 (H) and TUNEL (I) staining in tumor sections from mice with the indicated treatments (scale bar: 100 $\mu \mathrm{m})$. The data were presented as mean \pm s.d. Statistical analysis was performed using t test, $*, p<0.05$, $\star *, p<0.01, * * *, p<0.001$. 
A
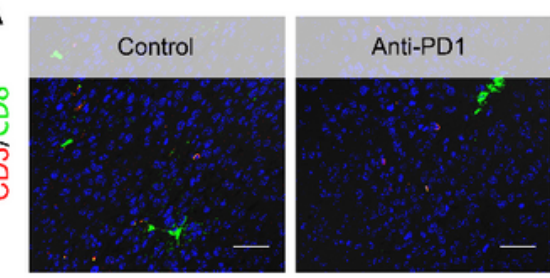

C
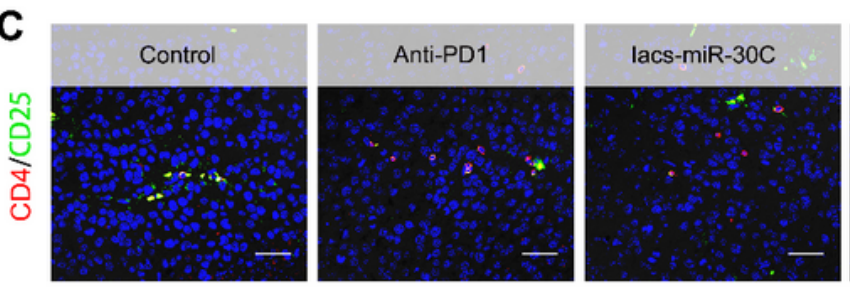

ki67

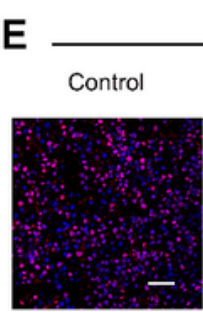

$E$
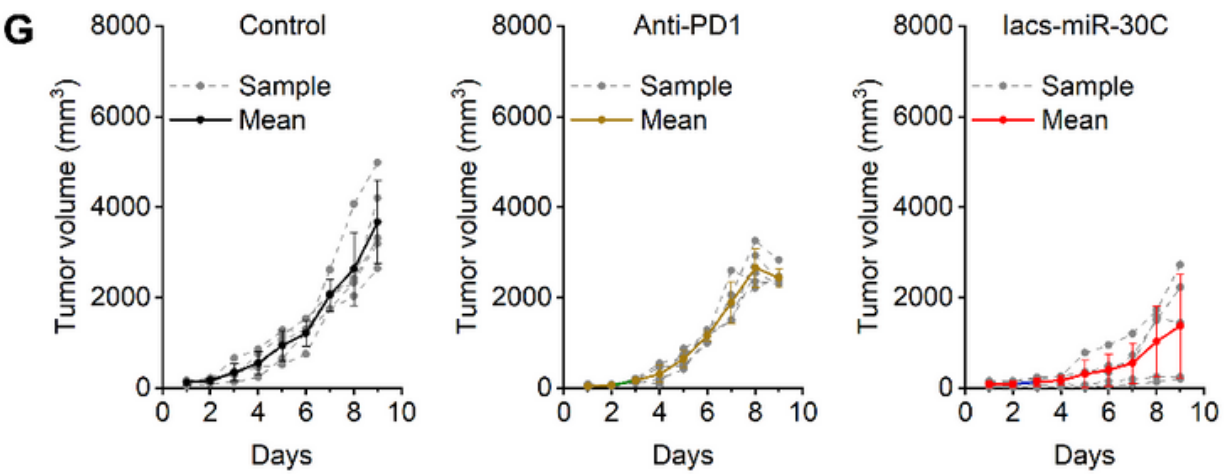

B
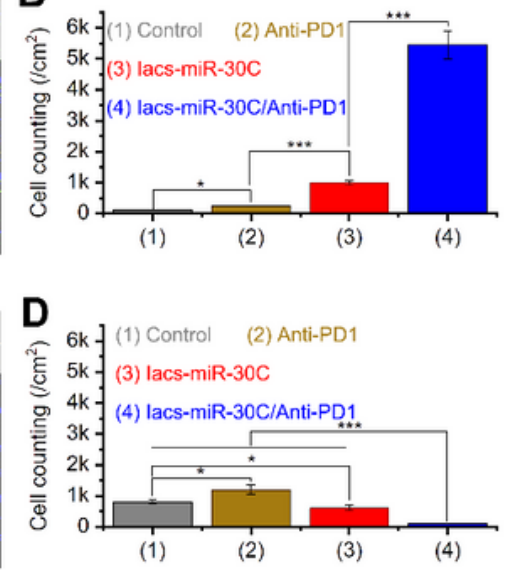

(1)

TUNEL
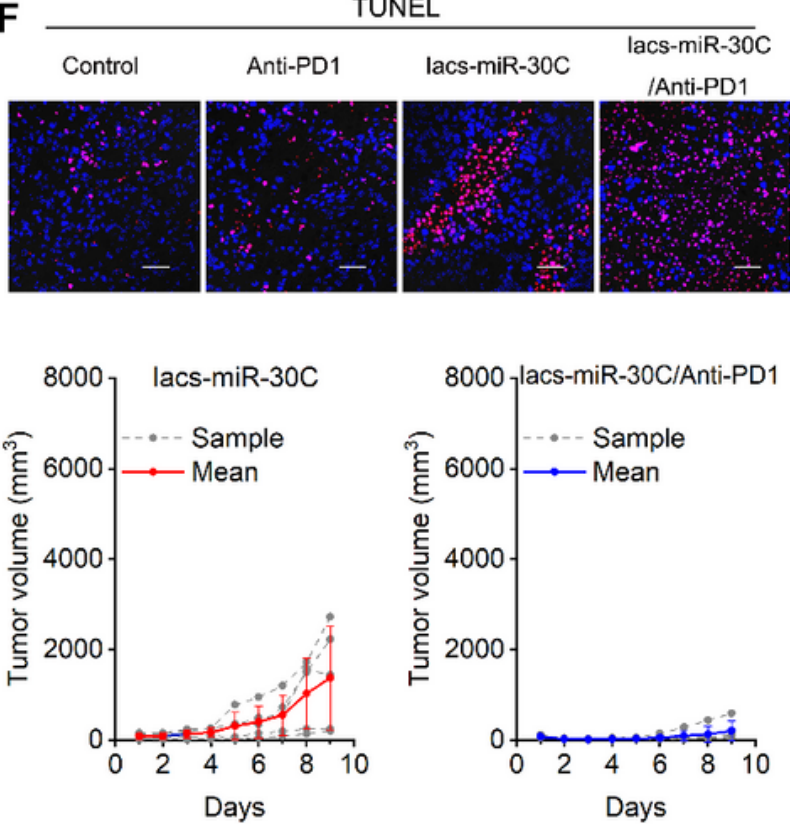
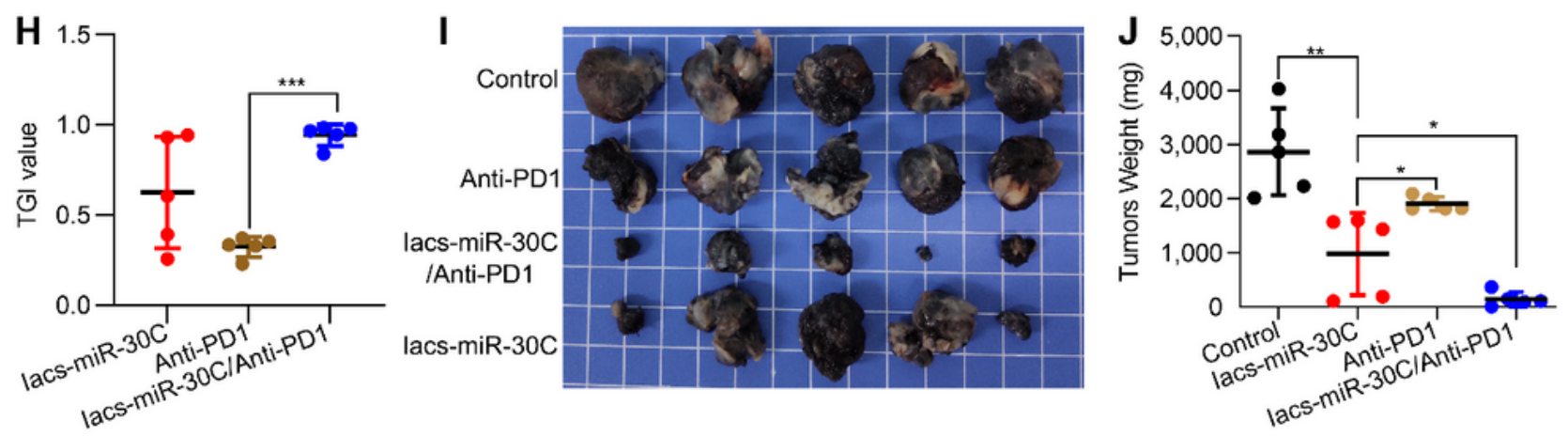

\section{Figure 6}

lacsRNA enhanceed anti-cancer activity of PD1 in C57 mice bearing B16F10 tumor. (A and B) Immunofluorescence images of $C D 3+/ C D 8+$ cells $(A)$ and quantification $(B)$ in tumor sections from mice with the indicated treatments (scale bar: $50 \mu \mathrm{m}$ ). (C and D) Immunofluorescence images of CD4+/CD25+ cells (C) and quantification (D) in tumor sections from mice with the indicated treatments (scale bar: 50 $\mu \mathrm{m})$. (E and F) Ki67 (E) and TUNEL (F) staining in tumor sections from mice with the indicated treatments 
(scale bar: $50 \mu \mathrm{m}) .(\mathrm{G})$ Growth curves of B16F10 tumors in C57 mice with the indicated treatments $(\mathrm{n}=$ 5). (H) Tumor growth inhibition (TGI) with the indicated treatment at day 9. (I and J) Representative photographs $(\mathrm{I})$ and weight of tumor $(\mathrm{J})$ after the indicated treatments. The data were presented as mean \pm s.d. Statistical analysis was performed using $t$ test, $*, p<0.05, * *, p<0.01, * \star *, p<0.001$.

\section{Supplementary Files}

This is a list of supplementary files associated with this preprint. Click to download.

- Graphicalabstractimage.tif

- Supplementary.docx 